\title{
Critical Phenomena in a Highly Constrained Classical Spin System: Néel Ordering from the Coulomb Phase
}

\author{
T. S. Pickles, T. E. Saunders, and J. T. Chalker \\ Theoretical Physics, University of Oxford, 1 Keble Road, Oxford, OX1 3NP, United Kingdom
}

(Dated: October 24, 2018)

\begin{abstract}
Many classical, geometrically frustrated antiferromagnets have macroscopically degenerate ground states. In a class of three-dimensional systems, the set of degenerate ground states has power-law correlations and is an example of a Coulomb phase. We investigate Néel ordering from such a Coulomb phase, induced by weak additional interactions that lift the degeneracy. We show that the critical point belongs to a universality class that is different from the one for the equivalent transition out of the paramagnetic phase, and that it is characterised by effective long-range interactions; alternatively, ordering may be discontinuous. We suggest that a transition of this type may be realised by applying uniaxial stress to a pyrochlore antiferromagnet.
\end{abstract}

PACS numbers: $\quad 64.60 . \mathrm{Cn}$ 75.10.Hk 05.70.Jk

The statistical physics of systems that combine local constraints with macroscopic degeneracy has been of enduring interest in several different contexts. Examples include models for geometrically frustrated antiferromagnets [1] and for proton disorder in ice [2], and dimer covering problems on a variety of lattices [3]. Local constraints arise from energy minimisation when ground states of a Hamiltonian are considered, or are rules that must be satisfied by all allowed configurations, as for closed-packed dimers on a lattice. Macroscopic degeneracy appears if there are extensively many ways to satisfy these constraints. Despite the degeneracy, correlation functions, averaged over states of this kind, need not be short-range. Indeed, it has recently been appreciated, both for dimer coverings of three-dimensional bipartite lattices, and for ground states of certain geometrically frustrated magnets, that pair correlations at large separation have a characteristic, algebraic dipolar form [4, 5, 6]. This behaviour is captured by the Coulomb phase of a theory in which the local constraints of lattice models become the requirement that a continuum field is solenoidal. The Coulomb phase is stable against all small perturbations that are allowed by symmetry, but a sufficiently large perturbation may induce long range order.

In this paper we consider a Néel ordering transition that takes place from the Coulomb phase of a geometrically frustrated antiferromagnet, asking how it differs from the conventional Néel transition out of the paramagnetic phase. Our interest in such a transition stems in part from the fact that, by design, it evades a naive application of Landau-Ginzburg-Wilson theory, since a description based in the standard way on symmetries and a local order parameter for the Néel phase will not reproduce the correlations of the Coulomb phase [7]. Similar considerations were also a motivation for an intriguing recent study of another ordering transition from a Coulomb phase, involving close-packed dimers on a cubic lattice 8]. In that case, high-precision Monte Carlo simulations indicate critical behaviour different from that expected at a conventional critical point with the same symmetries, but an analytical understanding of the transition has yet to be developed. By contrast, we show in the following how the Néel ordering transition from the Coulomb phase may be brought within the standard theoretical framework used to describe critical phenomena. More broadly, in light of the intense current interest in quantum phase transitions that lie beyond the scope of a conventional Landau treatment [9], it is natural to examine ordering from the Coulomb phase as a classical counterpart.
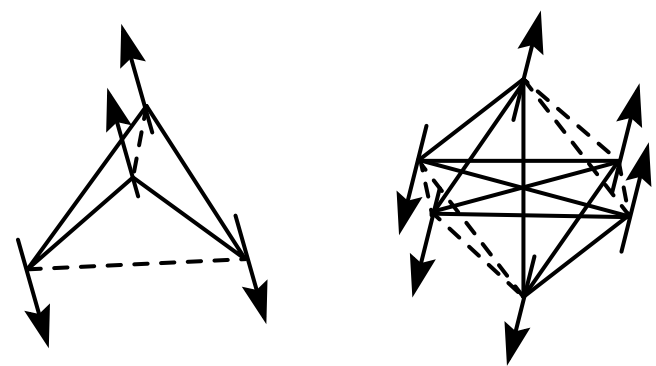

FIG. 1: Néel order induced by uniaxial compression in the building blocks of geometrically frustrated antiferromagnets. Left: a tetrahedron from the pyrochlore lattice strained along [100]. Right: an octahedron from a lattice of corner-sharing octahedra strained along [111]. Full lines denote antiferromagnetic exchange interactions of strength $J$, and dashed lines indicate ones of strength $J-\Delta$. Arrows represent spins.

The class of models we are concerned with is as follows. We study classical antiferromagnets on geometrically frustrated, three-dimensional lattices that are known to support a Coulomb phase in the zero temperature limit when all non-zero exchange interactions are the same. These systems meet three requirements: they have macroscopically degenerate ground states, do not display order by disorder, and have a bisimplex lattice. Such lattices consist of corner-sharing frustrated units, or simplices, which are arranged in a bipartite fashion, with exchange interactions linking all pairs of spins within each simplex. We focus on two examples: the Heisen- 
berg model on the pyrochlore lattice, built from cornersharing tetrahedra, which is approximately realised in a number of materials; and a system of $n$-component spins on a lattice of corner-sharing octahedra, which is a convenient theoretical construct. The local constraints satisfied in ground states of these models are that the total spin of each simplex is zero [10]. We induce order using a change in the relative strength of exchange interactions which can be produced by uniaxial compression via magnetoelastic coupling, as illustrated in Fig. 1. Exchange energies $J_{i j}$ within each simplex then take the two values $J$ and $J-\Delta$, and our focus is the regime $J \gg \Delta>0$. The Hamiltonian for both models has the form

$$
\mathcal{H}=\sum_{\langle i j\rangle} J_{i j} \mathbf{S}_{i} \cdot \mathbf{S}_{j}
$$

where $\mathbf{S}_{i}$ is an $n$-component unit vector. In each case, positive $\Delta$ removes the macroscopic ground state degeneracy of the unstrained system, selecting from these ground states the two-sublattice Néel states of Fig. 1] in which neighbouring pairs of spins coupled by the stronger interaction $J$ are antiferromagnetically aligned, while frustration forces those pairs coupled by the weaker interaction $J-\Delta$ to have ferromagnetic alignment. Considering the effect of thermal fluctuations, one expects Néel order below a critical temperature $T_{\mathrm{c}} \sim \Delta$, and a disordered phase at higher temperatures. For temperatures $T \ll J$, thermal fluctuations are predominantly within the ground state manifold of the unstrained system, and in the limit $J / \Delta \rightarrow \infty$ the transition at $T_{\mathrm{c}}$ is between a Néel phase and a Coulomb phase.

Several examples are known of magnetic ordering associated with lattice strain in frustrated magnets, although we are not aware of a direct realisation of the transition we discuss. In particular, Néel order has been induced by uniaxial stress in the pyrochlore $\mathrm{Tb}_{2} \mathrm{Ti}_{2} \mathrm{O}_{7}$ [11], while it is accompanied by spontaneous strain in the spinel $\mathrm{ZnCr}_{2} \mathrm{O}_{4}$ [12]. Unfortunately, $\mathrm{Tb}_{2} \mathrm{Ti}_{2} \mathrm{O}_{7}$ is a material that is at present poorly understood, and the transition in $\mathrm{ZnCr}_{2} \mathrm{O}_{4}$ is strongly first order. We hope that this paper will stimulate further experiments in the area.

We set out below several complementary approaches to understanding ordering in constrained systems. Starting from the continuum description of the Coulomb phase, Néel order amounts to condensation of the solenoidal field. When this transition is continuous, we show that it has the same critical behaviour as for an unconstrained system in which there are uniaxial dipolar interactions. From classic studies [13, 14, 15], it is known that longrange interactions of this type lead to an upper critical dimension of three, and we confirm this for the lattice models in the large- $n$ limit. We supplement this analysis with Monte Carlo simulations. We find that the transition in a Heisenberg model is first order, but show that a critical point can be reached by applying a staggered field.
We start by recalling the mapping of Refs. 5 and 6 , from ground states at $\Delta=0$ to solenoidal fields. The mapping uses the underlying simplex lattice for the spin system, which is a diamond lattice for the pyrochlore antiferromagnet, and a simple cubic lattice for the octahedral antiferromagnet. In each case, spins $\mathbf{S}_{i}$ are located at mid-points of bonds $i$ on the simplex lattice. Unit vectors $\hat{\mathbf{e}}_{i}$ are defined on these bonds, with the orientation convention that they are all directed from a given simplex sublattice towards the other one. A spin configuration is represented using $n$ flavours of a three-component field, defined from the spin components $S_{i}^{\alpha}$ by $\mathbf{B}_{i}^{\alpha}=S_{i}^{\alpha} \hat{\mathbf{e}}_{i}$. The ground state constraint, that the total spin of each simplex is zero, translates into the condition that these fields have lattice divergence zero. Introducing continuum coordinates $\mathbf{r}$ and coarse-grained fields $\mathbf{B}^{\alpha}(\mathbf{r})$ satisfying $\nabla \cdot \mathbf{B}^{\alpha}(\mathbf{r})=0$, the entropic weight on ground states is postulated [5, 6] to be proportional to $e^{-\mathcal{H}_{0}}$, where

$$
\mathcal{H}_{0}=\frac{\kappa}{2} \int \mathrm{d}^{3} \mathbf{r} \sum_{\alpha}\left|\mathbf{B}^{\alpha}(\mathbf{r})\right|^{2}
$$

The stiffness $\kappa$ is the single parameter of the theory: it simply controls the amplitude of correlations.

Under the same mapping, the Néel states of Fig. 1 are represented by a uniform, non-zero $\mathbf{B}^{\alpha}(\mathbf{r})$. The orientation of this field in real space is along [100] for the pyrochlore antiferromagnet, and along [111] for the octahedral antiferromagnet, while the orientation in flavour space reflects the $\mathrm{O}(n)$ symmetry-breaking of the Néel order.

In order to describe a phase transition between the Coulomb and Néel phases, we incorporate the effect of non-zero $\Delta$ into the continuum theory of Eq. (2) via a temperature-dependent control parameter $t$, with $t=1$ for $T \gg \Delta$ and $t<0$ for $T \ll \Delta$. Separating $\mathbf{B}^{\alpha}(\mathbf{r})$ into components $B_{\|}^{\alpha}(\mathbf{r})$ and $\mathbf{B}_{\perp}^{\alpha}(\mathbf{r})$, respectively parallel and perpendicular to the preferred direction, we replace $\mathcal{H}_{0}$ by $\mathcal{H}_{2}+\mathcal{H}_{4}$, where

$$
\begin{gathered}
\mathcal{H}_{2}=\frac{\kappa}{2} \int \mathrm{d}^{3} \mathbf{r} \sum_{\alpha}\left\{\left|\mathbf{B}_{\perp}^{\alpha}(\mathbf{r})\right|^{2}+t\left|B_{\|}^{\alpha}(\mathbf{r})\right|^{2}+\left|\nabla \times \mathbf{B}^{\alpha}(\mathbf{r})\right|^{2}\right\} \\
\mathcal{H}_{4}=u \int \mathrm{d}^{3} \mathbf{r}\left\{\sum_{\alpha}\left|\mathbf{B}^{\alpha}(\mathbf{r})\right|^{2}\right\}^{2} .
\end{gathered}
$$

At $t=1$, Eq. (3) is simply $\mathcal{H}_{0}$ supplemented by gradient and quartic terms that are irrelevant in the scaling sense in the Coulomb phase. On reducing $t$ a transition occurs (at $t=0$ within mean field theory) to an ordered phase in which $\left\langle B_{\|}^{\alpha}(\mathbf{r})\right\rangle \neq 0$. The quartic term $\mathcal{H}_{4}$ is required for stability in the ordered phase, while the gradient term is necessary to suppress short-wavelength fluctuations close to the critical point.

The solenoidal constraint can be re-expressed as a longrange interaction. To this end, write the fields $\mathbf{B}^{\alpha}(\mathbf{r})$ in terms of vector potentials $\mathbf{A}^{\alpha}(\mathbf{r})$ by setting $\mathbf{B}^{\alpha}(\mathbf{r})=$ 
$\nabla \times \mathbf{A}^{\alpha}(\mathbf{r})$, and choose the Coulomb gauge $\nabla \cdot \mathbf{A}^{\alpha}(\mathbf{r})=0$. Then $\mathcal{H}_{2}$ is diagonalised by Fourier transform and by rotation in the space of the three components of $\mathbf{A}^{\alpha}$. In a fixed gauge only two of these components fluctuate independently: taking their amplitudes at wavevector $\mathbf{q}$ to be $a_{1}^{\alpha}(\mathbf{q})$ and $a_{2}^{\alpha}(\mathbf{q})$, the diagonalised form is

$$
\begin{aligned}
& \mathcal{H}_{2}=\frac{\kappa}{2} \int d^{3} \mathbf{q} \sum_{\alpha}\left\{\left[q_{\|}^{2}+t q_{\perp}^{2}+q^{4}\right] a_{1}^{\alpha}(-\mathbf{q}) a_{1}^{\alpha}(\mathbf{q})\right. \\
&\left.+\left[q^{2}+q^{4}\right] a_{2}^{\alpha}(-\mathbf{q}) a_{2}^{\alpha}(\mathbf{q})\right\} .
\end{aligned}
$$

To emphasise the equivalence to a system with uniaxial dipolar interactions, we rescale the fields by a factor $q$, writing $\varphi_{l}^{\alpha}(\mathbf{q})=q a_{l}^{\alpha}(\mathbf{q})$ for $l=1,2$; and since $\varphi_{2}^{\alpha}(\mathbf{q})$ is non-critical, we omit it, obtaining finally

$$
\mathcal{H}_{2}=\frac{\kappa}{2} \int d^{3} \mathbf{q} \sum_{\alpha}\left[(1-t) \frac{q_{\|}^{2}}{q^{2}}+t+q^{2}\right] \varphi_{1}^{\alpha}(-\mathbf{q}) \varphi_{1}^{\alpha}(\mathbf{q}) .
$$

In this form, $\mathcal{H}_{2}$ has been studied extensively as a model for uniaxial ferroelectrics and ferromagnets, with the term $q_{\|}^{2} / q^{2}$, non-analytic at $q=0$, arising from dipolar interactions. Ordering in these systems is celebrated as an example of a phase transition with an upper critical dimension of three, at which critical behaviour is almost mean field, but with logarithmic corrections [13, 14, 15]. We conclude that the same behaviour will appear at the Néel ordering transitions of the constrained spin systems discussed here, a demonstration of the controlling influence that the correlations of the Coulomb phase have on critical phenomena.

We next turn to lattice models, and first present an alternative way of viewing the equivalence between the constrained spin systems we study and ones with uniaxial dipolar interactions. Consider the the spectrum of the interaction matrix $J_{i j}$ appearing in Eq. (11). It is block-diagonalised by Fourier transform with wavevector k. In the class of models we consider, at $\Delta=0$ its lowest branch is dispersionless, and for bisimplex lattices this branch is degenerate with dispersive branches at points $\mathbf{k}_{\mathrm{D}}$ in the Brillouin zone. A natural approach to statistical mechanics at $T \ll J$ is via projection onto these flat bands. The projection operator $P$ is non-analytic in $\mathbf{k}$ at $\mathbf{k}_{\mathrm{D}}$ and as a result is power-law in real space at large distances [16]. For three-dimensional, bisimplex frustrated antiferromagnets, its long-distance form is dipolar [17].

The dipolar nature of $P$, applied to certain pyrochlore magnets (spin-ice materials [18]) turns out to have the remarkable consequence that dipolar interactions are essentially equivalent to nearest-neighbour ones, within the set of low-energy states allowed by local constraints, a feature termed projective equivalence [17]. Here we take an opposite path: we show that projection of $J_{i j}$ for $\Delta>0$ turns nearest-neighbour interactions into longrange ones.
On favouring Néel order by setting $\Delta>0$, degeneracy in the spectrum of $J_{i j}$ is lifted, leaving isolated minima. In this language, the models of Fig. 1 1 are engineered so that these minima occur at the degeneracy points $\mathbf{k}_{\mathrm{D}}$. Then for $\Delta \ll J$ the interaction matrix inherits the singularities of the projection operator. To be definite, we now focus on the octahedral lattice, for which $\mathbf{k}_{\mathrm{D}}=(\pi, \pi, \pi)$; we have also obtained equivalent results for the pyrochlore lattice. Introducing $\mathbf{q}=\mathbf{k}-\mathbf{k}_{\pi}$, the projected interaction has a lowest eigenvalue that varies with $\mathbf{q}$ as

$$
\mathcal{E}(\mathbf{q})=\mathcal{E}_{0}+3 \Delta \frac{q_{\|}^{2}}{q^{2}}+\mathcal{O}\left(q^{2}\right),
$$

where $q_{\|}$is the component of $\mathbf{q}$ in the [111] direction and $\mathcal{E}_{0}$ is a constant. Spin configurations that are thermally allowed for $T / J \rightarrow 0$ are invariant under projection: the presence in Eq. (6) of the singular term $q_{\|}^{2} / q^{2}$ is therefore a key result, indicating effective uniaxial dipolar interactions within these configurations.

With $T / J$ small but non-zero, the power-law correlations of the Coulomb phase are cut off at a large distance $\xi_{\mathrm{C}}$. In these circumstances, uniaxial dipolar interactions will control critical phenomena provided the correlation length $\xi$ is smaller than $\xi_{\mathrm{C}}$, but with a crossover to conventional behaviour sufficiently close to $T_{\mathrm{c}}$.

To study the phase transition in the lattice model, we use the large- $n$ limit, extending the treatment of geometrically frustrated systems described in [5, 19], and recovering the critical behaviour known for uniaxial dipolar systems at $n \rightarrow \infty[13,14,15]$. As a function of reduced temperature $t_{\mathrm{r}}$, we find that the staggered susceptibility $\chi$ diverges as $\chi \sim\left|t_{\mathrm{r}}\right|^{-1} \cdot \ln \left(1 /\left|t_{\mathrm{r}}\right|\right)$, and the sublattice magnetisation vanishes as $m \sim\left|t_{\mathrm{r}}\right|^{1 / 2}$.

To examine these ideas further, we use Monte Carlo simulations. We study the pyrochlore Heisenberg antiferromagnet, treated extensively at $\Delta=0$ in previous work [10]. Crucially, the absence of energy barriers in this model at $\Delta=0$ makes it possible to equilibrate simulations at temperatures as low as $T=10^{-4} \mathrm{~J}$, permitting access to the regime we are concerned with. We focus on the two choices: $\Delta=0.1 J$ and $\Delta=10^{-3} J$, using systems of $N=4 L^{3}$ spins, with $N \leq 4000(L \leq 10)$ and employing parallel tempering [20].

For $\Delta=0.1 J$, we find (data not shown) a continuous transition at $T_{c}=0.267 \mathrm{~J}$ and critical behaviour consistent with the standard, short-range universality class, which is unsurprising since $\xi_{\mathrm{C}}$ is not large at this temperature $\left(\xi_{\mathrm{C}} \propto(J / T)^{1 / 2}\right.$ for $n \geq 2$ and $\left.T \ll J\right)$. For $\Delta=10^{-3} J$ the data illustrated in Fig. 2 indicate a strongly first-order transition. The Néel order parameter shows a rapid change with temperature, and this becomes more step-like with increasing system size. Moreover, close to the transition the probability distribution for the energy develops two peaks, a feature characteristic of a first-order transition [21]. 


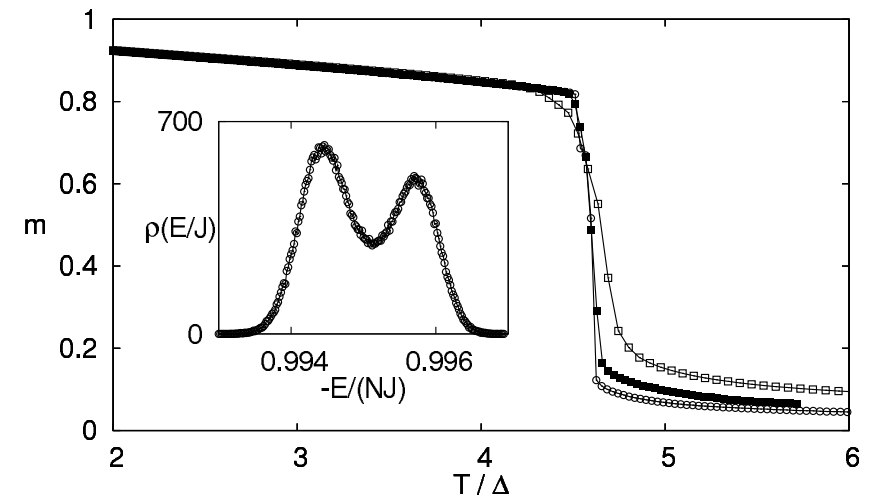

FIG. 2: Evidence for a first-order transition from simulations with $\Delta=10^{-3} J$. Main panel: $m$ vs. $T / J$ for $L=6(\square)$, $8(\boldsymbol{\square})$, and $10(\circ)$. Inset: probability distribution $\rho(E / J)$ for the energy $E$ at the estimated transition temperature, $T / J=4.6 \times 10^{-3}$, for $L=6$.

There is an obvious interest in finding a version of this ordering transition which is continuous even at $\Delta \ll J$, as a realisation of the uniaxial critical behaviour we have discussed. It is possible that the order of the transition could be altered by a change in microscopic details of the system. Here, however, we follow an alternative and more automatic route to a critical point, by applying a staggered field: we add to Eq. (11) the term $-h \sum_{i} n_{i} S_{i}^{z}$, with $n_{i}= \pm 1$ taking opposite signs on the two Néel sublattices. For $h \ll \Delta$, a first-order transition survives, between a high-temperature phase with small sublattice magnetisation $m$ and a low-temperature one with large $m$. Increasing $h$, the discontinuity in $m$ at the transition is reduced, and above a critical field $h_{\mathrm{c}} \sim \Delta$ there is no thermally driven phase transition. The critical end point at $h=h_{\mathrm{c}}$ is the continuous transition we seek. Evidence for its existence is presented in Fig 3 , A precise numerical study of critical behaviour at $h=h_{\mathrm{c}}$ for $\Delta / J \ll 1$ would be challenging and we do not attempt it here, but we believe the theoretical case for uniaxial dipolar critical behaviour at this transition is compelling. We note that, while the staggered field used in our simulations cannot be realised in a Heisenberg system, it would arise from a uniform external field in spin-ice materials [18].

In summary, we have shown that ordering in highly constrained spin systems can be quite different from its counterpart in conventional systems. We suggest that experiments on ordering in uniaxially strained geometrically frustrated magnets should be of great interest.

We thank L. Balents, R. Moessner, V. Pasquier, and particularly P. C. W. Holdsworth, for helpful discussions. This work was supported by EPSRC Grant No. GR/R83712/01. Part of it was done while JTC was a visitor at KITP Santa Barbara, supported by NSF Grant No. PHY99-07949.

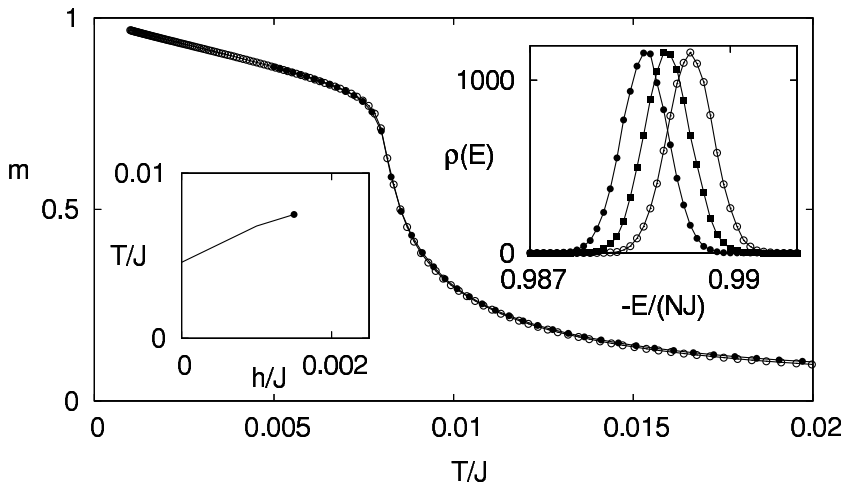

FIG. 3: Transition in a staggered field with $\Delta=10^{-3} J$. Left inset: Schematic phase diagram, showing first-order transition line terminating at a critical end point. Main panel: smooth variation of $m$ with $T$ at $h=2 \times 10^{-3} \mathrm{~J}$, which is greater than $h_{\mathrm{c}}$, for $L=6$ (०) and $L=8(\bullet)$. Right inset: probability distribution $\rho(E / J)$ for the energy $E$ at $T / J=8.0 \times 10^{-3}, 8.3 \times 10^{-3}$ and $8.6 \times 10^{-3}$, showing single peaks.

[1] P. W. Anderson, Phys. Rev. 102, 1008 (1956).

[2] L. Pauling, J. Am. Chem. Soc. 57, 2680 (1935).

[3] R. H. Fowler and G.S. Rushbrooke, Trans. Faraday Soc. 33, 1272 (1937).

[4] D. A. Huse et al. Phys. Rev. Lett. 91, 167004 (2003).

[5] S. V. Isakov et al. Phys. Rev. Lett. 93, 167204 (2004).

[6] C. L. Henley, Phys. Rev. B 71, 014424 (2005).

[7] D. L. Bergman, G. A. Fiete, and L. Balents, Phys. Rev. B 73, 134402 (2006).

[8] F. Alet et al., Phys. Rev. Lett. 97, 030403 (2006).

[9] T. Senthil et al., Science 303, 1490 (2004).

[10] R. Moessner and J. T. Chalker, Phys. Rev. Lett. 80, 2929 (1998); Phys. Rev. B 58, 12049 (1998).

[11] L. Mirebeau et al., Nature 420, 54 (2002); Phys. Rev. Lett. 93, 187204 (2004).

[12] S.-H. Lee et al., Phys. Rev. Lett. 84, 3718, (2000).

[13] A. I. Larkin and D. E. Khmel'nitskii, Sov. Phys. JETP 29, 1123 (1969).

[14] A. Aharony, Phys. Rev. B 8, 3363 (1973).

[15] E. Brezin and J. Zinn-Justin, Phys. Rev. B 13, 251 (1976).

[16] J. Des Cloizeau, Phys. Rev. 135, A698 (1964).

[17] S. V. Isakov, R. Moessner, and S. L. Sondhi, Phys. Rev. Lett. 95, 217201 (2005).

[18] S. T. Bramwell and M. J. P. Gingras, Science 294, 1495 (2001).

[19] B. Canals and D. A. Garanin, Can. J. Phys. 79, 1323 (2001).

[20] K. Hukushima and K. Nemoto, J. Phys. Soc. Japan, 65, 1604 (1996); for a review, see: E. Marinari in Advances in Computer Simulation, edited by J. Kertész and I. Kondor (Springer-Verlag, Berlin, 1998).

[21] K. Binder, Rep. Prog. Phys. 50, 783 (1987). 